\title{
A Hybrid Data-Driven Online Solar Energy Disaggregation System from the Grid Supply Point
}

This paper was downloaded from TechRxiv (https://www.techrxiv.org).

LICENSE

CC BY-NC-SA 4.0

SUBMISSION DATE / POSTED DATE

04-09-2021 / 09-09-2021

\section{CITATION}

Zhang, Xiao-Yu; Watkins, Chris; Kuenzel, Stefanie (2021): A Hybrid Data-Driven Online Solar Energy Disaggregation System from the Grid Supply Point. TechRxiv. Preprint. https://doi.org/10.36227/techrxiv.16569744.v1

$\mathrm{DOI}$

10.36227/techrxiv.16569744.v1 


\title{
A Hybrid Data-Driven Online Solar Energy Disaggregation System from the Grid Supply Point
}

\author{
Xiao-Yu Zhang, Student Member, IEEE, Chris Watkins, Lei Yin, Stefanie Kuenzel, Senior Member, \\ IEEE
}

\begin{abstract}
The integration of small-scale PV systems (such as roof-top PVs) decreases the visibility of the power system since the real demand load is masked. Most of the rooftop systems are behind-the-meter and cannot be measured by the household smart meter. To overcome the challenges mentioned above, this paper proposes an online solar energy disaggregation system to decouple the solar energy generated by the roof-top PV systems and ground truth demand load from the net measurements. A 1D CNN bidirectional long short-term memory (CNN-BiLSTM) deep learning method is used as the core algorithm of the proposed system. The system takes a wide range of online information (AMI data, meteorological data, satellite-driven irradiance, and temporal information) as inputs to evaluate the PV generation, and the system also enables online and offline modes. The effectiveness of the proposed algorithm is evaluated by comparing it to baselines. The results show that the proposed method reaches good performance under different penetration rates and different feeder levels. Finally, a transfer learning process is introduced to verify the proposed system has good robustness and can be applied to anywhere else easily.
\end{abstract}

Index Terms - Behind-the-meter solar generation, rooftop PV, deep neural network, transfer learning, energy disaggregation, data-driven analysis.

\section{INTRODUCTION}

\section{A. Motivation}

THE global cumulative PV capacity has increased from 480 1 GW in 2018 to $583.5 \mathrm{GW}$ by the end of 2019 , representing a $21 \%$ growth over the year before $[1,2]$. Among all newly installed capacities, rooftop PV (residential PV) increases 16 $\mathrm{GW}$, occupies 15\% of all solar that was installed in 2019. Although the high PV penetration reduces greenhouse gas emissions and leads to an environmental-friendly world, it also changes the existing power system structure significantly. One significant problem, the California "Duck Curve", is caused by the high penetration of sustainable energy (solar energy, wind energy, etc.) into the net load, a significant drop is observed during midday, causing the unbalance between demand and supply. It is vital to increase the visibility of these renewable energy generations to better manage the power system. Among all installed solar panels, small-scale PV or rooftop PV occupies nearly $50 \%$ of the overall capacity. Unlike the large-scale PV stations that be measured individually, most rooftop PV is behind-the-meter, which means the power generated by PV

X-Y. Zhang and S. Kuenzel are with the Department of Electronic Engineering, Royal Holloway, University of London, TW20 0EX, U.K (email: Xiaoyu.Zhang.2018@live.rhul.ac.uk).

C. Watkins is with the Department of Computer Science, Royal Holloway, University of London, TW20 0EX, U.K

L. Yin is with State Grid Huainan Electric Power Supply Company, China. cannot be recorded by the residential smart meter. Lack of visibility would limit the demand-side management, schedule the short-term operation implemented by the grid operators. The conventional method requires installing an electricity meter beside the solar panel of each house, which requires extra measurement devices as well as communication channels. These devices largely increase budgets and invade personal privacy [3]. Moreover, the ownership of these devices also raises conflicts among stakeholders.

\section{B. Problem Statement}

The feeder system is shown in Fig. 1, where the feeder connects a few houses and unmonitored rooftop PV systems. The power utility has the authority to access real-time grid measurement of a feeder/substation (active power $P_{N e t}(t)$, reactive power $Q_{N e t}(t)$, voltage $V_{N e t}(t)$, current $I_{N e t}(t)$ etc.). The components under the feeder include the total demand load of all residences served by the feeder, $P_{\text {Load }}$, and power generated by the rooftop PVs in this area, $P_{P V}$ :

$$
P_{\text {Net }}(t)=P_{\text {Load }}(t)-P_{P V}(t)
$$

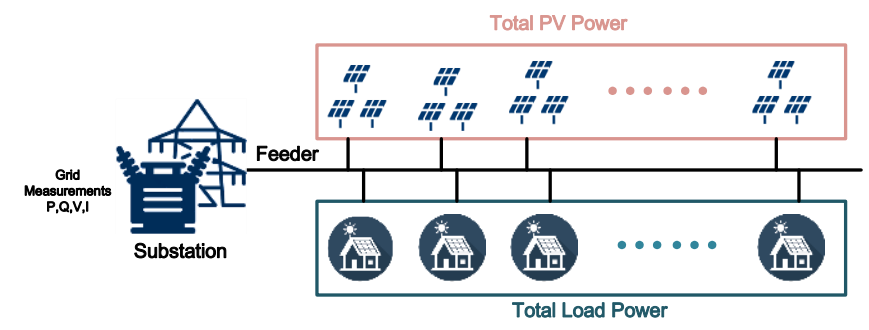

Fig. 1. Power system with a PV system installed along the feeder.

The target of the proposed system is to decouple $P_{\text {Net }}(t)$ into $P_{\text {Load }}(t)$ and $P_{P V}(t)$, as shown in Fig. 2. It is observed that the penetration of solar energy distorts the original demand load curve, making it is difficult to recover the original demand load from the masked net load.

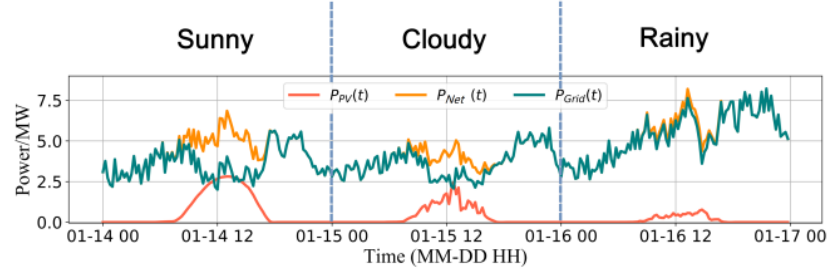

Fig. 2. Example of time series $P_{P V}(t), P_{N e t}(t), P_{\text {Load }}(t)$ under different weather conditions (Data source: Pecan Street Dataport [4]).

\section{Related work}

Methods to decouple solar energy and the demand load are discussed in the literature. These methods can be divided into 
three categories: model-based method, upscaling method, and data-driven method.

The model-based method estimates the total solar energy in a region via constructing an equivalent PV system [5-10]. Taking grid measurements, weather information, and irradiance information as inputs, the parameters of the equivalent system are optimized by solving an optimization problem. Few regression methods are proposed, including Contextually Supervised Source Separation (CSSS) methodology [9] and its extension[10], convex optimization [5, 7], and multiple support vector regression models [4]. In addition, ambient temperature is also adopted as a correction factor to improve the model accuracy in some works [7, 10]. In [4], K. Li et.al proposed an Ensemble model by considering sub-models under different weather conditions, the capacity of the PV systems is also estimated automatically. To sum up, the model-based method requires knowledge of the PV module model (angle of solar radiation, series resistance, etc.). Lacking vital information would lead to a large error between ground truth and estimation.

Upscaling method [11-14] selects a small number of representing PV systems as the reference to estimate the overall PV generation of the whole area. In [11, 12], a method that combines a data dimension reduction procedure and a mapping function (linear regression, Kalman filter) are proposed. Moreover, a hybrid method that combines upscaling with satellite-derived is proposed in $[13,14]$. Next-generation highresolution satellites produce high accurate irradiance estimates that can improve performance. Upscaling methods require measurements from a small group of PV systems in the target area, this information is not always available for rooftop PV.

To overcome the shortages of approaches mentioned above, the data-driven method utilizes high-resolution data from a variety of data resources that correlate to solar energy to estimate solar energy [11,12,14-17]. The power data comes from micro-phasor measurement units ( $\mu$ PMUs), supervisory control, and SCADA, and smart meters. While meteorological data comes from National Centres for Environmental Information (NCEI) (US) [18], Climatological Observers Link (COL) (UK) [19], and satellite data from The National Solar Radiation Database (NSRDB) (US) [25], or RE Data Explorer (Central Asia) [26]. F. Bu et.al. [15] proposed a game-theoretic approach, a closed-loop game-theoretic approach is used to search the optimal composite exemplars from a candidate library, and a semi-supervised source separator is employed for disaggregation. In [16], Y. Wang et.al. proposed a hybrid method that combines the model-based method and data-driven load/PV forecasting techniques. The PV generation and demand load are decoupled at first and each component is forecasted individually via neural network and gradient boosting regression tree (GBRT). Moreover, a Multi-layer perceptron (MLP) neural network is raised in [17], measurement data from various sources are feed into the network to implement the disaggregation. The results show that the hybrid model achieves better performance than models that only utilize one data source.

To sum up, method-based methods have a strong mathematical explanation of the PV systems, but lacking adaptation and flexibility is strictly limited. While upscaling method is suitable for large-scale PV systems, and data-driven methods heavily rely on data resources and do not have strong mathematical definitions. The proposed hybrid approach takes advantage of both the model-based approach and data-driven approach, which is described as follows.

\section{Contributions}

To overcome the drawbacks of existing approaches, a solar energy disaggregation system that enables both online and offline modes is proposed in this paper. Instead of installing measurement devices at each house, the proposed method only utilizes measurements from one smart meter installed in the feeder or substation to estimate the solar energy generation in the whole area. Other relevant variables, such as meteorological, irradiance, and temporal data are also collected as the inputs of the disaggregation system. The main novelties of this paper are as follows:

1) A CNN-BiLSTM based solar energy disaggregation system that enables online and offline modes. Instead of installing a meter to each individual rooftop PV, the proposed system can decouple the PV generation and real demand load of a geographical area by only installing a feeder/substationlevel smart meter.

2) Comparing to traditional bidirectional LSTM that can only enable offline learning, an online BiLSTM algorithm that uses a rolling window is adopted.

3) A case study demonstrates that the proposed disaggregation system achieves high accuracy under different solar energy penetration rates and different feeder models.

4) A transductive transfer learning approach that utilizes synthetic data to evaluate real-time generation at other substations or feeders.

\section{E. Organization of the Paper}

The resulting paper is organized as follows: The background is introduced in Section II. The feature extraction and data preprocessing approach are demonstrated in Section III. In section IV, the solar energy disaggregation model, as well as algorithms, are proposed. The case studies are presented in Section V. The conclusion and final discussion are drawn in the last section.

\section{Feature Extraction and Data Processing}

In this section, the feature extraction and data processing methods are introduced. The selection of features that correlate to solar energy generation is vital to the efficiency of the model. Moreover, to improve data quality and remove significant noise, the data should be pre-processed before feeding data into the model. Pre-processing steps include data cleansing (remove duplicate observations, fill in missing data, etc.), feature scaling (normalization, standardization, etc.), and dimensionality reduction.

\section{A. Features Selection}

Apart from the grid measurements which indicate the variation of load and PV generation, other features such as satellite-driven irradiance features, temporal-related features, and meteorological features also influence solar energy 
generation. Fig. 3 shows the Pearson Correlation Coefficients of PV output and relevant numerical variables. When the coefficient is greater than 0.3 , a positive correlation exists between the two variables. From the figure, it is observed that among all variables, temperature, GHI, DNI, DHI, and cloud cover rate have the most impact on the PV output. The detailed feature extraction is shown as follow:

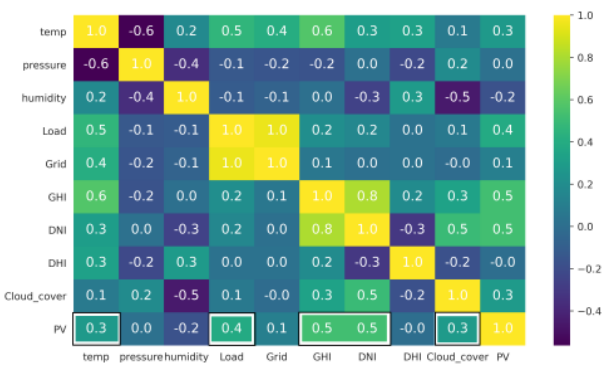

Fig. 3. Correlation coefficients of PV generation and relevant variables (Data source: Pecan Street Dataport [4], NCDC [20], NCEI [18]).

1) Grid Measurement: By accessing the grid measurements provided by the feeder-level smart meter/SCADA, features such as active/ reactive power, voltage, current are collected. In this paper, active power $P_{\text {grid }}$, reactive power $Q_{\text {grid }}$ are chosen as the grid measurement variables. The duration of peak PV generation (10 am to $15 \mathrm{pm})$ and peak load is different (7am to $10 \mathrm{am}$, and $17 \mathrm{pm}$ to $22 \mathrm{pm}$ ), by using the variation of the load curve as the input feature, the overall net load can be decoupled into the real load curve and PV generation.

2) Satellite-driven Irradiance Features: The irradiance measurements and weather data at the same location are obtained from the National Climatic Data Center (NCDC) [20]. Satellite-driven data include GHI, DNI, DHI, latitude, longitude, etc.

- GHI: The total amount of shortwave radiation received from above by a surface horizontal to the ground.

$$
G H I=D N I \cos (\theta)+D H I
$$

- DNI: Amount of solar radiation received per unit area by a surface that is always held perpendicular (or normal) to the rays that come in a straight line from the direction of the sun at its current position in the sky.

- DHI: The amount of radiation received per unit area by a surface (not subject to any shade or shadow) that does not arrive on a direct path from the sun but has been scattered by molecules and particles in the atmosphere and comes equally from all directions.

Fig.4 shows the heatmap of GHI and PV output throughout the whole year, respectively. From the figure, it is found that the PV output correlates to the GHI value strongly. The duration of the peak values of GHI/PV output almost overlapping.

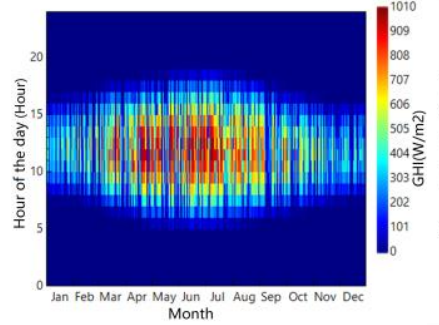

(a)

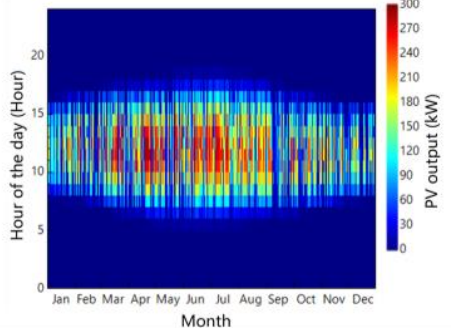

(b)
Fig. 4. Heatmap of (a) GHI/ (b) PV output throughout the whole year (Data source: NCDC [20]).
3) Temporal-Related Features: The temporal variables include the number of the hour of the day $H$, the month of the year $M$, and the quarter of the year $R$. Examples of average PV outputs and probability density distributions during different months are presented in Fig. 4. It is observed that both the month and the quarter of the year influence the PV outputs. Normally, the maximum output throughout the year appears between June to August.

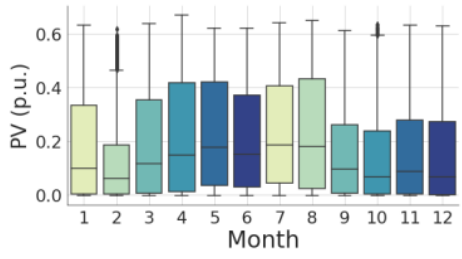

(a)

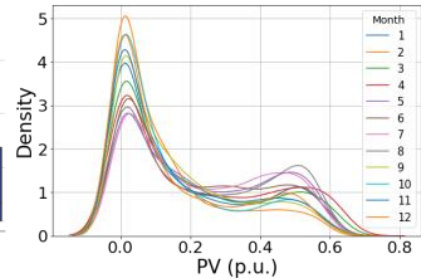

(b)
Fig. 5. (a) Bar chart of PV outputs in different months (b) probability density distributions during different months (Data source: Pecan Street Dataport [4]).

4) Meteorological Features: Meteorological Data is collected by NCEI [18], features include temperature $T$, humidity $U$, weather condition (e.g., sunny, rainy, snowy, cloudy) $W$, cloud cover rate $D$, surface albedo, pressure, wind speed, etc. From Fig.3, it is observed that $T, U, D$ are correlated to the solar energy, while other variables have week or negative correlation. Fig.6 makes a comparison of average PV output referring to different weather conditions, it is observed that the output power reaches a maximum during clear days, and less power is produced during bad weather conditions such as rainy and snowy. Hence, the weather condition is also a vital variable in this case.

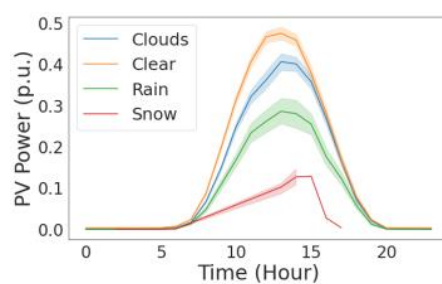

(a)

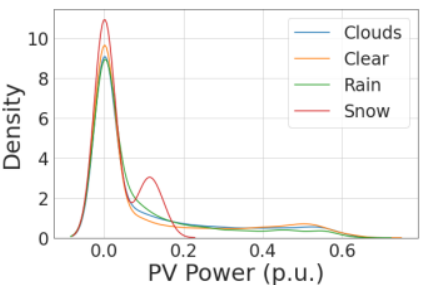

(b)
Fig. 6. (a) PV output under different weather conditions; (b) probability density distributions under different weather conditions (Data source: Pecan Street Dataport [4]).

To summarize, all features can be divided into numerical variables $\mathbf{N}$ and categorical variables $\mathbf{C}$. The numerical variables $\mathbf{N}$ is:

$$
\boldsymbol{N}_{\boldsymbol{t}}=\left[P_{\text {grid }, t}, Q_{\text {grid }, t}, T_{t}, U_{t}, D_{t}, G H I_{t}, D N I_{t}, D H I_{t}\right]
$$

\section{B. One-Hot Encoder for Transformation}

A categorical variable is a category or type. Although some categorical variables are recorded as a number, they do not have numerical meaning, such as month and hour. Before feeding the data to the DNN model, all categorical variables should be converted to numerical forms via one-hot encoding. A new binary variable is used to represent the original variable [21,22]. In this paper, categorical variable matrix $\boldsymbol{C}$ contains:

$$
\boldsymbol{C}_{\boldsymbol{t}}=\left[W_{t}, H_{t}, M_{t}, R_{t}\right]
$$

By implementing one-hot encoding, the variables are transferred to:

$$
\boldsymbol{C}_{\boldsymbol{t}}^{o}=f^{O}\left(\boldsymbol{C}_{\boldsymbol{t}}\right)
$$


where $f^{O}$ is the one-hot encoding function, and $\boldsymbol{C}_{\boldsymbol{t}}^{\boldsymbol{O}}$ is the onehot encoding matrix. Hence, the overall input matrix $\boldsymbol{D}$ is shown as follows:

$$
D_{t}=\left[N_{t}, C_{t}^{O}\right]
$$

\section{Data Dimension Reduction (PCA)}

As introduced in previous sections, there are many features that should be taken into consideration in this paper. Too many features will slow down the computation speed and difficult to find an optimal solution. Principal Component Analysis or PCA is a common technique to reduce the dimensionality of large datasets which contain a variety of variables [22, 23]. The purpose of PCA is to transfer the dataset with $n$ dimensions down to $d$ dimensions $(d<n)$, meanwhile most useful information is preserved by selecting the axis that preserves the maximum amount of variance.

As shown in Algorithm 1, Considering the dataset which is a $m \times n$ centralized data matrix ( $m$ is the number of samples, $\mathrm{n}$ is the number of features). By decomposing the covariance matrix of data, eigenvectors and eigenvalues are obtained. Ranking the eigenvalues from largest to smallest, the eigenvectors corresponding to $d$ largest eigenvalues are selected as the new basis vectors (principal components). Finally, the original $n$ dimension dataset is projected to $d$ dimensions.

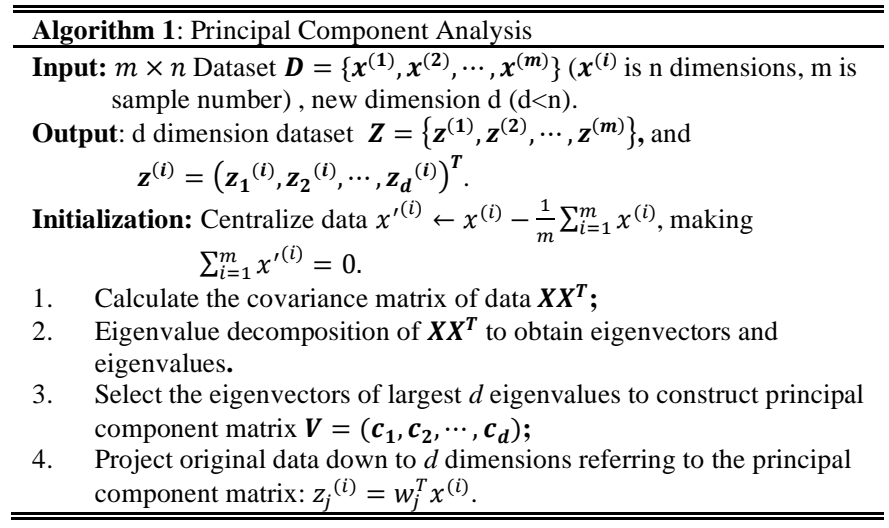

\section{DeEP Learning Algorithms}

\section{A. Algorithms for Comparison}

1) Gradient Boosting Algorithm: Gradient Boosting algorithm is a kind of machine learning algorithm that produces a prediction model from a series of weak prediction models [24]. Normally, the Gradient Boosting algorithm contains three elements: a differentiable loss function for optimization, a squared error is adopted as the loss function for the regression task; a weak prediction model to make a prediction, a decision tree is used as a weak model in Gradient Boosting; and an additive model that can add all weak models together and minimize the losses.

2) Multilayer Perceptron (MLP): X. Zhang et.al [17] proposes an MLP based PV desegregation scheme. As a type of feedforward neural network, MLP is constructed with multiple layers of perceptron with activation functions. A typical MLP consists of one input layer, one output layer, and several hidden layers.

3) $1 D$ Convolutional Neural Network ( $1 D C N N)$ : $1 \mathrm{D} C \mathrm{CNN}$ is similar to 2D/3D CNN but with a different input structure. In $1 \mathrm{D} \mathrm{CNN}$, the kernel moves along one dimension, and the shapes of input and output data are both two dimensions. 1D CNN is extremely suitable for processing 1D data (such as time-series data, text, and audio). Kernel size represents the width of the kernel, while the height of the kernel is the amount of data at each step.

4) LSTM algorithm: The structure of the LSTM block is illustrated since LSTM has been the basic component of the BiLSTM model. The structure of a typical LSTM block is shown in Fig. 7, the cell state $c_{t}$ is different from the output $h_{t}$. As demonstrated, the components inside the block include an input node $g_{t}$, input gate $i_{t}$, internal gate $c_{t}$, forget gate $f_{t}$, output gate $o_{t}$, and output $h_{t}$. The nature of the gate is a sigmoid unit (output range between $[0,1]$ ), it can recognize and pass important information and block unimportant information. Once both input and output gates are closed, the flow will be blocked inside the memory cell and will not affect the following time steps until the gate reopens. As indicated in state-space equation (7), both $g_{t}, i_{t}, f_{t}$ and $o_{t}$ are functions of data in current time step input $x_{t}$ as well as the output of the previous time step $h_{t-1}$. and $b_{i}, b_{f}, b_{o}$ are bias parameters of nodes. After the value of gates have been determined, the candidate value $\tilde{c}_{t}$ is calculated and compared with the previous cell state $c_{t-1}$. With the gate status $i_{t}$ and $f_{t}$, the memory cell determines whether update its value or not. Finally, by regulating the current cell state $c_{t}$ with tanh activation function $\phi$ and multiplying with output gate, the output value is calculated:

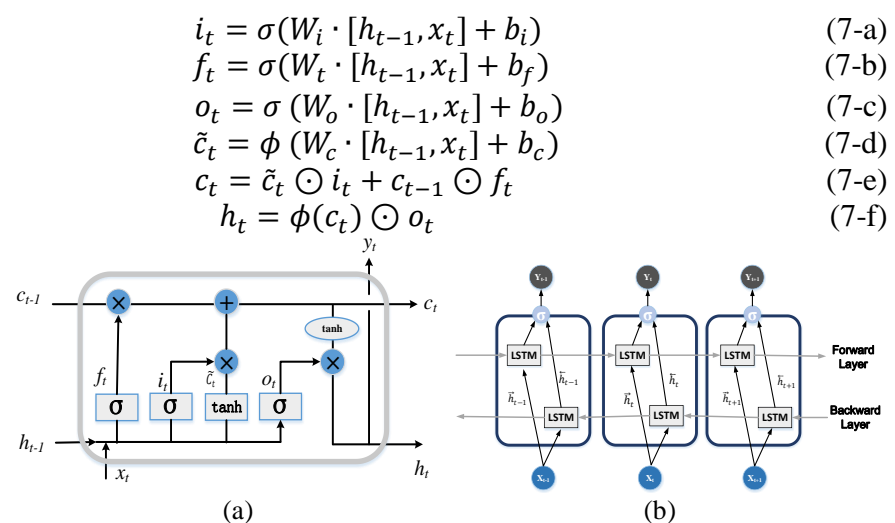

Fig. 7. The structure of (a) LSTM block; (b) Bidirectional LSTM block.

\section{B. Hybrid CNN Bidirectional LSTM}

1D CNN-BiLSTM is a combination of Bidirectional LSTM (BiLSTM) RNN with one dimensional CNN, providing deeper learning ability for regression tasks with time-series data [22].

The chain-like structure and memory cell help RNN learn temporal dynamic characteristics of time series data. LSTM, as a typical RNN, is widely adopted in forecasting and speech recognition tasks. While conventional LSTM can only pass information in a positive direction (from previous time steps to current timestamp), BiLSTM enables information to flow both 
forward and backward, helping the neural network better understand the characteristics of the data to be learned.

\section{Disaggregation System Models}

\section{A. Overall System}

The proposed system is assumed to have the authority to access real-time grid measurement by the AMI/ SCADA. And the system can also get measurements from weather stations and satellites. The target of the system is to separate the net load into the demand load and PV generation. The proposed system enables two operation modes: online and offline modes, see Fig. 8 . The online mode can provide PV disaggregation on a realtime basis, and the off-line mode implements algorithms with a historical database.

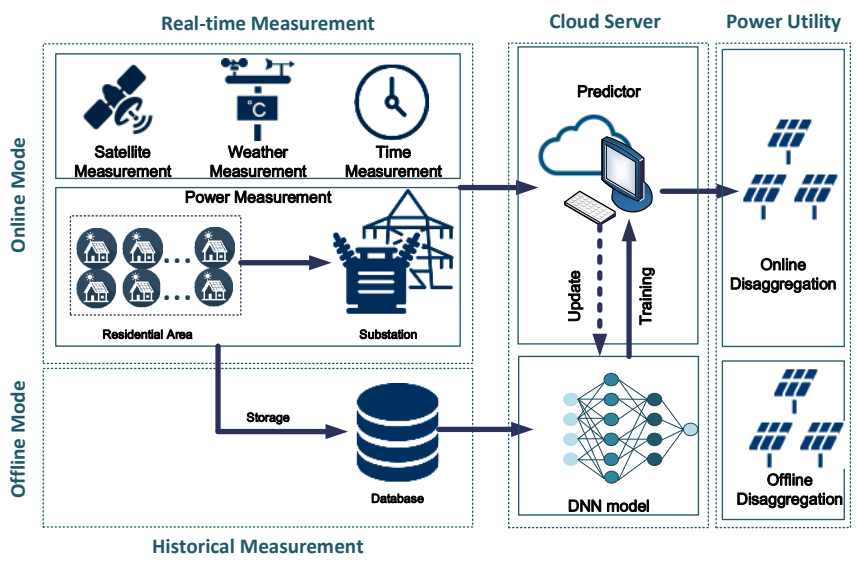

Fig. 8. Online/Offline PV energy disaggregation framework.

1) Online mode: The online mode part of the system consists of three components: real-time measurement, cloud server, and power utility. In online mode, the PV disaggregation system can access real-time measurements from both distribution feeders as well as weather stations/satellite systems. Grid measurements include active power, reactive power, voltage, current, etc. Weather-related measurements include temperature, humidity, cloud cover, etc. After data is gathered from the sensors/stations, the data is pre-processed, and the dimensionality of the data is reduced via PCA. The dimensionreduced data is then fed into the core part of the system, the cloud platform which implements deep learning algorithms (introduced in the next subsection) to decouple the original net load into PV generation and real demand load.

Conventional bidirectional LSTM cannot support online learning due to the delay problem. This is because, during the online mode, we assume the length of the input sequence is unknown and it is impossible to learn the input sequence from both forward and backward directions. Hence, an online BiLSTM algorithm is adopted which is originally used for online speech recognition [25]. A sliding window moves over the real-time input sequence, then the BLSTM is implemented for each sliding window, in this case, the model can learn bidirectionally, and the time delay reduces to $T_{w}$. The side window with size $T_{w}$ is moving with a time step $T_{s}$, so the original online sequence can be split into a few chunks, and the $i$-th window is:

$$
\boldsymbol{Z}_{i}=\left[z_{i T_{S}+1}, z_{i T_{S}+2}, \ldots, z_{i T_{S}+T_{W}}\right]
$$

and a maximum number of $T_{w} / T_{s}$ windows overlap at a time stamp $t$. The final output at time stamp $t$ is evaluated by averaging the output of overlapping windows at $t$. It is noticed the online system results in a delay of $T_{w}$, since the system should lookup $T_{w}-1$ timestamp in the future to determine the output at time stamp $t$.

2) Offline mode: Real-time measurements are not always available to the power utility; offline mode is adopted to analyse the load components based on historical data. Historical data are generated from SCADA and relevant data centre and stored in the database. In offline mode, the input sequence is provided by the database and conventional BiLSTM is adopted since the whole input sequence is available. The offline mode does not require real-time communication with SCADA or weather stations but decouples the historical grid measurements into solar energy and demand load locally. Offline mode can help the power utility construct the local PV and load model, contributing to the demand-side management effort.

After the disaggregation system was introduced, four machine learning/ deep learning algorithms are proposed to implement disaggregation, which are Gradient Boosting algorithm, MLP, One-dimensional CNN (1D CNN), and CNN BiLSTM.

\section{Performance eValuation}

\section{A. Data Description}

1) Feeder model: Standard feeder models provided by GridLAB-D are listed in Table I [26]. The feeder types are classified depending on the residence description, ranging from light rural to moderate urban (with apparent power from 948 $\mathrm{kW}$ to $17021 \mathrm{~kW}$ ). There are two methods to construct a feederlevel dataset: (1) Add the data of houses together to match the value of feeder power. (2) Use simulation software to simulate the feeder.

TABLE I

SUMMARY OF PROTOTYPICAL FEEDERS [26]

\begin{tabular}{llll}
\hline \hline Feeder & $\begin{array}{l}\text { Rated } \\
\text { voltage/kV }\end{array}$ & $\begin{array}{l}\text { Rated } \\
\text { power/kW }\end{array}$ & Description \\
\hline R1-12.47-4 & 12.47 & 5334 & Heavy suburban \\
R2-25.00-1 & 24.9 & 17021 & Moderate urban \\
R4-25.00-1 & 24.9 & 948 & Light rural \\
\hline \hline
\end{tabular}

2) Load and PV data: Pecan Street Dataport [4] is used as the dataset to train the proposed model. The dataset contains one thousand household-level power consumption in the last five years with an interval resolution of 15 minutes. In this paper, one interval resolution data is adopted. The household-level measurements are added together to construct a synthetic feeder model. In this paper, 75 houses are aggregated to build a feeder with a capacity of $100 \mathrm{~kW}$ during Jan 2018 and Dec 2018 in Austin, Texas, US. The PV penetration rate of the feeder is adjusted via adding or deleting houses with PV installed to match the requirement.

3) Meteorological and Irradiance Dataset: Meteorological Dataset from NCEI [18] given specific location and duration is adopted, the same from irradiance dataset provided by NCDC.

\section{B. Software\& Hardware}

The simulation and computation are implemented on a Dell laptop equipped with a Core i7-7700HQ CPU, NVIDIA GTX 
$1060 \mathrm{GPU}$, and 8GB RAM. The deep learning algorithm runs on Python 3.6, the TensorFlow framework is adopted to train the DNN model.

\section{Experimental Setup}

In this section, three case studies are implemented to investigate how penetration rate, hyperparameters influence the performance of proposed disaggregation algorithms. Moreover, the data transferability is studied to investigate whether a model can be pre-trained with a synthetic dataset.

\section{Evaluation Criteria}

To evaluate the performance of proposed disaggregation algorithms, three evaluation metrics are adopted, which are Root Mean Squared Error (RMSE), Normalized RMSE (nRMSE), and R-squared $\left(\mathrm{R}^{2}\right)$. Detailed metrics are shown as follows:

\section{1) Root Mean Squared Error (RMSE)}

$$
R M S E=\sqrt{\frac{\sum_{t=1}^{T}\left(\hat{P}_{P V, t}-P_{P V, t}\right)}{T}}
$$

2) Normalized RMSE (nRMSE)

$$
n R M S E=\frac{R M S E}{\bar{P}_{P V}}
$$

3) Mean Absolute Percentage Error (MAPE):

4) $R$ Square $\left(R^{2}\right)$

$$
M A P E=\frac{\sum_{i=1}^{N}\left|\left(y_{i}-\hat{y}_{i}\right) / y_{i}\right|}{N} \times 100 \%
$$

$$
R^{2}=\frac{\sum_{i=N}^{N}\left(t_{i}-\bar{t}\right)\left(y_{i}-\bar{y}\right)}{\sqrt{\sum_{i=1}^{N}\left(t_{i}-\bar{t}\right)^{2} \sum_{i=1}^{N}\left(y_{i}-\bar{y}\right)^{2}}}
$$

\section{Results AND Discussion}

\section{A. Case study I: Performance under Different PV \\ Penetration Rate}

In this case study, the proposed CNN-BiLSTM algorithm is compared to state-of-the-art deep learning/ machine learning algorithms (MLP, LSTM, and Gradient boosting (GB)). Three feeder models described in Table I with different PV penetration rates (ranging from 5\% to 20\%) are studied. In net load, the PV penetration rate is defined as the percentage of PV output compared to the peak demand load:

$$
P V \text { peneration }(\alpha)=\frac{\text { Peak } P V \text { Load }}{\text { peak demand load }}
$$

The simulation results are shown in Table II. From the table, it is observed that the proposed CNN-BiLSTM performances best among all disaggregation algorithms for all three feeder models. However, in some cases, such as the case with $\alpha=5 \%$ in the Heavy Suburban feeder model, LSTM has less nRMSE value and higher $R^{2}$ value, compared to the CNN-BiLSTM method. Moreover, the MLP method is the worst method to disaggregate solar energy from the demand load since it has the

\begin{tabular}{|c|c|c|c|c|c|}
\hline & MAE (kW) & 5.86 & 6.40 & 5.24 & 5.10 \\
\hline & nRMSE (\%) & 11.68 & 8.22 & 11.29 & 6.68 \\
\hline & RMSE (kW) & 11.07 & 7.79 & 10.70 & 6.33 \\
\hline \multirow{4}{*}{20} & $\mathrm{R}^{2}$ & 0.81 & 0.86 & 0.79 & 0.93 \\
\hline & MAE $(\mathrm{kW})$ & 11.01 & 12.51 & 10.73 & 9.55 \\
\hline & nRMSE (\%) & 10.82 & 8.86 & 11.32 & 6.82 \\
\hline & RMSE (kW) & 20.52 & 16.80 & 21.46 & 12.94 \\
\hline \multicolumn{6}{|c|}{ Heavy Suburban } \\
\hline \multirow{4}{*}{5} & $\mathrm{R}^{2}$ & 0.79 & 0.93 & 0.81 & 0.91 \\
\hline & MAE (kW) & 16.54 & 16.06 & 16.22 & 13.90 \\
\hline & nRMSE (\%) & 11.36 & 6.33 & 10.70 & 7.15 \\
\hline & RMSE (kW) & 30.31 & 16.88 & 28.53 & 19.06 \\
\hline \multirow{4}{*}{10} & $\mathrm{R}^{2}$ & 0.78 & 0.90 & 0.86 & 0.94 \\
\hline & MAE (kW) & 31.38 & 33.37 & 28.76 & 26.91 \\
\hline & nRMSE (\%) & 11.14 & 7.149 & 9.16 & 5.88 \\
\hline & RMSE (kW) & 59.44 & 38.11 & 48.86 & 31.38 \\
\hline \multirow{4}{*}{20} & $\mathrm{R}^{2}$ & 0.80 & 0.91 & 0.83 & 0.93 \\
\hline & MAE (kW) & 58.53 & 43.08 & 51.64 & 40.23 \\
\hline & nRMSE (\%) & 10.85 & 6.40 & 9.99 & 6.33 \\
\hline & RMSE (kW) & 115.82 & 68.24 & 106.62 & 67.53 \\
\hline \multicolumn{6}{|c|}{ Moderate Urban } \\
\hline \multirow{4}{*}{5} & $\mathrm{R}^{2}$ & 0.79 & 0.88 & 0.82 & 0.88 \\
\hline & MAE (kW) & 49.48 & 49.65 & 42.79 & 42.48 \\
\hline & nRMSE (\%) & 11.12 & 8.07 & 10.35 & 7.97 \\
\hline & RMSE (kW) & 94.62 & 68.64 & 88.10 & 67.83 \\
\hline \multirow{4}{*}{10} & $\mathrm{R}^{2}$ & 0.79 & 0.89 & 0.81 & 0.91 \\
\hline & MAE (kW) & 101.49 & 119.93 & 89.62 & 89.95 \\
\hline & nRMSE (\%) & 11.23 & 7.97 & 10.75 & 7.20 \\
\hline & RMSE (kW) & 191.07 & 135.67 & 183.03 & 122.48 \\
\hline \multirow{4}{*}{20} & $\mathrm{R}^{2}$ & 0.80 & 0.88 & 0.77 & 0.93 \\
\hline & MAE (kW) & 188.69 & 265.95 & 200.29 & 179.88 \\
\hline & nRMSE2 (\%) & 10.85 & 7.87 & 11.61 & 6.00 \\
\hline & RMSE (kW) & 369.37 & 267.82 & 395.27 & 204.28 \\
\hline
\end{tabular}
highest nRMSE and MAE in all cases.

$$
\text { TABLE II }
$$

DisAGgREgation PERFORMANCE UNDER DifFERENT PENETRATION RATE

\begin{tabular}{llllll}
\hline \hline $\boldsymbol{\alpha}(\boldsymbol{\%})$ & Metrics & GB & LSTM & MLP & $\begin{array}{l}\text { CNN- } \\
\text { BiLSTM }\end{array}$ \\
\hline \multicolumn{5}{c}{ Light Rural } \\
\hline \multirow{3}{*}{5} & $\mathrm{R}^{2}$ & 0.79 & 0.89 & 0.80 & $\mathbf{0 . 9 2}$ \\
& MAE $(\mathrm{kW})$ & 2.91 & 2.92 & 2.66 & $\mathbf{2 . 5 2}$ \\
& nRMSE $(\%)$ & 11.67 & 7.95 & 11.27 & $\mathbf{7 . 0 5}$ \\
& RMSE $(\mathrm{kW})$ & 5.53 & 3.77 & 5.34 & $\mathbf{3 . 3 4}$ \\
\hline 10 & $\mathrm{R}^{2}$ & 0.79 & 0.88 & 0.80 & $\mathbf{0 . 9 4}$ \\
\hline
\end{tabular}

The other observation from Table II is the performance under different PV penetration rates. It is shown that the performance of the proposed CNN-BiLSTM improves with the increase of $\alpha$, the value of $R^{2}$ increases from $0.88-0.92$ with $\alpha=5 \%$ to 0.93 0.94 when $\alpha=20 \%$.

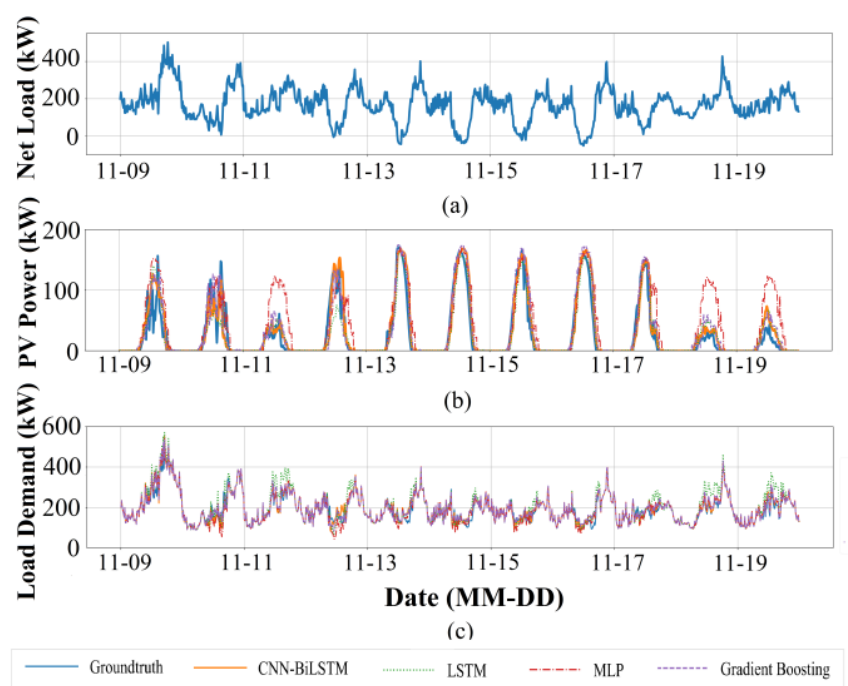

Fig. 9. Decoupling performance for the feeder with $\bar{P}_{L}=948 \mathrm{KW}$, and $\alpha=20 \%$.

Fig. 9 shows an example of the decoupling performances of all four disaggregation algorithms, as well as the ground truth value, for the feeder with $\overline{\mathrm{P}}_{\mathrm{L}}=948 \mathrm{KW}$, and $\alpha=20 \%$. In the top plot, the original net load curve is presented, compared to the net load without PV systems, a dramatic drop is observed between 8:00 am to 17:00 pm, which is a typical characteristic to identify the PV generations. The middle plot shows the estimations of PV outputs performed by four algorithms, while the bottom plot shows the decoupled demand load curve. The figure shows that although PV outputs inferred by all 
algorithms closely match the ground truth curve, the proposed CNN-BiLSTM presents the best estimation. In this case, the $\mathrm{R}^{2}$ for CNN-BiLSTM is around 0.93, and nRMSE is 6.82\%. Moreover, a reduction of $39.75 \%$ in nRMSE value compared to the MLP algorithm.

Several estimation examples under different weather conditions are presented in Fig. 10. Three weather conditions: clear sky, overcast, and rain. Three typical days are given for each weather condition category. From the figure, it is found that all four algorithms track the ground truth curve very well in clear sky days. While on rainy and overcast days, since the sun is covered intermittently, these algorithms cannot always catch up with the actual PV outputs. Poor performance is observed in the case of the MLP algorithm, as a huge error is detected between the actual curve and the estimated curve. When turns to the proposed CNN-BiLSTM, which is the orange solid line in Figure 10, it can track the groudtruth PV outputs precisely even during rain and overcast days.

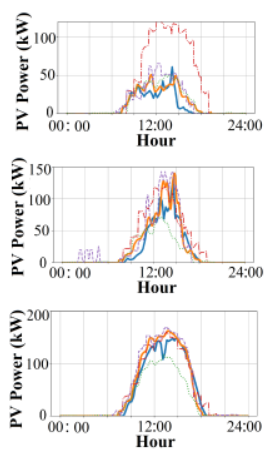

Day 1
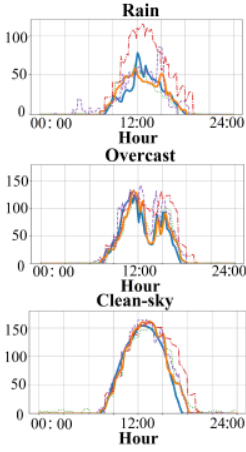

Day 2
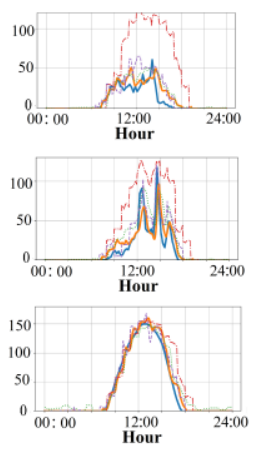

Day 3
Groundtruth $\longrightarrow$ CNN-BiLSTM ….... LSTM -

Fig. 10. Examples of the estimation results of four disaggregation algorithms under different weather conditions (sunny, rainy, cloudy).

\section{B. Case Study II: Transductive Transfer Learning}

In our previous case study, the training and testing data is used consistently from the same dataset. However, it is difficult to obtain labeled data at the target location. To overcome the limitation, which forms a major hurdle for real word industrial applications, a transductive transfer learning approach is proposed. The definition of transfer learning is defined as follows:

Definition 1 (Transfer Learning) [27]. Providing a source domain $\mathcal{D}_{s}$ and learning task $\mathcal{T}_{S}$, a target domain $\mathcal{D}_{T}$ and learning task $\mathcal{T}_{T}$, the purpose of transfer learning is to help improve the performance of the target function $\mathcal{F}_{T}$ in $\mathcal{D}_{T}$ using the knowledge in $\mathcal{D}_{S}$ and $\mathcal{T}_{S}$, where $\mathcal{D}_{S} \neq \mathcal{D}_{T}$, or $\mathcal{T}_{S} \neq \mathcal{T}_{T}$.

While in a transductive transfer learning task, source learning task $\mathcal{T}_{S}$ and target learning task $\mathcal{T}_{T}$ are the same (to implement decoupling task), but the domains of source and target may be different $\left(\mathcal{T}_{S}\right.$ is a synthetic dataset, and $\mathcal{T}_{T}$ is real-time data in this case)[28]. In this paper, the transductive transfer learning framework can be split into four steps (see Fig.11):

- Step 1 - Synthetic Database Generation: Referring to the local conditions (such as load capacity, geographical information, the portion of PV tilt angles, meteorological data, etc. ), simulation software is adopted to generate synthetic solar energy and demand load dataset. Hence, the dataset is labelled and can be used for supervised learning.

- Step 2-Data Analysis: As introduced in Section III, the generated dataset is pre-processed to provide normalized, featured extracted data.

- Step 3 - Model Generation: With processed data, the CNN-BLSTM neural network model is trained, and the model parameters are stored in a cloud server.

- Step 4-Transfer Learning: The unlabelled real-time data from the target area, is then sent to the trained model, while the cloud server would decouple the net load into solar energy and demand load.

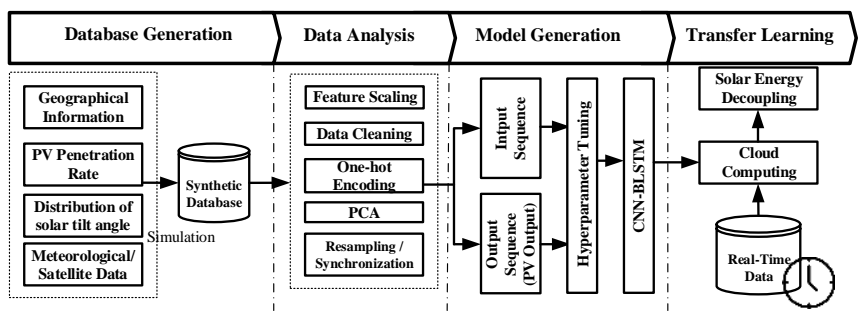

Fig. 11. Block diagram of the transfer learning process.

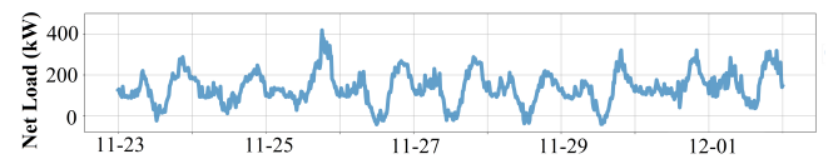

(a)

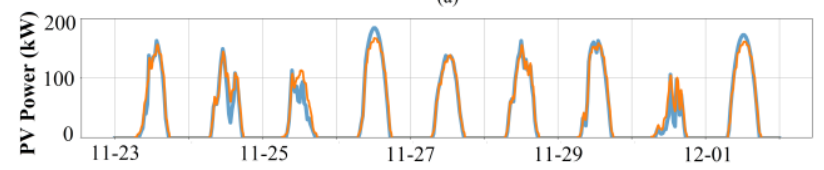

(b)

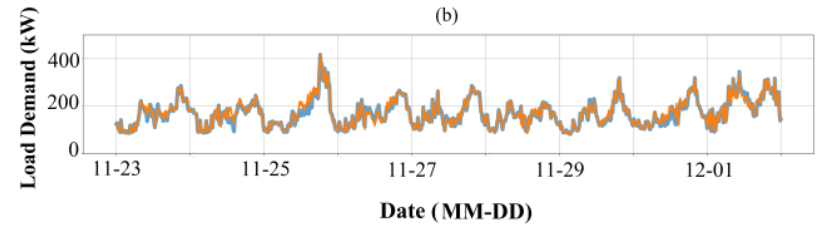

(c)

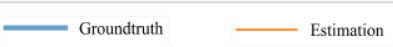

Fig. 12. The performance of the transfer learning in Austin, Texas, US.

TABLE III

RELEVANT INFORMATION ABOUT THE TARGET AREA

\begin{tabular}{llllll}
\hline \hline Case & Location & Year & $\begin{array}{l}\text { Load Capacity } \\
(\mathbf{k W})\end{array}$ & $\begin{array}{l}\text { Penetration } \\
\text { Rate (\%) }\end{array}$ & $\begin{array}{l}\text { Optimal Tilt } \\
\text { Angle }\left({ }^{\circ}\right)\end{array}$ \\
\hline $\mathbf{1}$ & Austin & 2018 & 948 & 20 & 28 \\
$\mathbf{2}$ & New York & 2019 & 17021 & 5 & 34 \\
\hline \hline
\end{tabular}

In the simulation, two cases are studied to investigate the proposed transfer learning method in Austin, Texas, and New York, detailed description of the cases is shown in Table III. SAM simulation software [29] is adopted to generate a synthetic residential solar energy dataset referring to the given relevant information. The data provided by Dataport is adopted as real-time measurements. The performance of the disaggregation system in transfer learning is presented in Fig. 12 and Table IV. The CNN-BiLSTM model is pre-trained via 
the synthetic dataset and then applied to the aggregated realmeasured demand load. In Case 1, where the target area is selected in Austin, Texas, the transfer learning almost researches the equal performance in Case study I; as for Case 2, where the target area is selected in New York, the performance is slightly worse than the model which is trained via realmeasured dataset. This is because there is a minor geographical information error between the location of the synthetic data and real-measured sites. The two cases show that the proposed transfer learning method is easy to implement anywhere else and a desirable accuracy can be achieved.

TABLE IV

RELEVANT INFORMATION ABOUT THE TARGET AREA

\begin{tabular}{lllll}
\hline \hline Cases & $\mathbf{R}^{\mathbf{2}}$ & MAE $(\mathbf{k W})$ & nRMSE2 (\%) & RMSE $(\mathbf{k W})$ \\
\hline 1 & 0.96 & 5.03 & 5.14 & 9.74 \\
2 & 0.79 & 72.19 & 3.70 & 125.94 \\
\hline \hline
\end{tabular}

\section{CONCLUSION}

This paper develops a deep learning-based solar energy disaggregation system to decouple the solar energy generated by the rooftop PV systems and real demand load from the net load measured by the feeder level smart meter. The system collects various information from different resources as inputs, including AMI data, meteorological data, satellite-driven irradiance, and temporal information. A 1D CNN bidirectional LSTM algorithm is developed to estimate the solar energy generated in the target area. Compared to benchmark algorithms (Gradient Boosting, MLP, and naïve LSTM), the precision and effectiveness of the proposed method are verified via several case studies. Both the influence of the PV penetration rate as well as feeder load capacity on the proposed system are fully investigated. The results show that the proposed method can decouple solar energy with a low error even at a low penetration rate (5\%). Moreover, the method is robust since it can be adapted to different feeder models and the model can be trained via a synthetic dataset and still achieve desirable performance in real-world measurement. These characteristics enable the proposed system to be widely adopted and practical in implementation. In future work, this study can be extended to take energy storage systems and other renewable energy into consideration. In addition, an unsupervised learning algorithm is to be developed to simplify the training process.

\section{REFERENCES}

[1] G. E. C. IRENA, "Renewable capacity statistics 2020," International renewable energy agency, 2020.

[2] M. DrIncecco, S. Squartini, and M. Zhong, "Transfer Learning for NonIntrusive Load Monitoring," IEEE Transactions on Smart Grid, vol. PP, pp. 1-1, 08/28, 2019.

[3] X. Y. Zhang, S. Kuenzel, J.-R. Córdoba-Pachón, and C. Watkins, "Privacy-Functionality Trade-Off: A Privacy-Preserving Multi-Channel Smart Metering System," Energies, vol. 13, 2020.

[4] P. Street, "Dataport: the world's largest energy data resource," Pecan Street Inc, 2015.

[5] C. M. Cheung, W. Zhong, C. Xiong, A. Srivastava, R. Kannan, and V. K. Prasanna, "Behind-the-meter solar generation disaggregation using consumer mixture models," in 2018 IEEE International Conference on Communications, Control, and Computing Technologies for Smart Grids (SmartGridComm), 2018, pp. 1-6.

[6] E. C. Kara, C. M. Roberts, M. Tabone, L. Alvarez, D. S. Callaway, and E. M. Stewart, "Disaggregating solar generation from feeder-level measurements," Sustainable Energy, Grids Networks, vol. 13, pp. 112$121,2018$.
[7] F. Sossan, L. Nespoli, V. Medici, and M. Paolone, "Unsupervised Disaggregation of PhotoVoltaic Production from Composite Power Flow Measurements of Heterogeneous Prosumers," IEEE Transactions on Industrial Informatics, vol. PP, 06/15, 2017.

[8] K. Li, F. Wang, Z. Mi, M. Fotuhi-Firuzabad, N. Duić, and T. Wang, "Capacity and output power estimation approach of individual behindthe-meter distributed photovoltaic system for demand response baseline estimation," Applied Energy, vol. 253, pp. 113595, 2019.

[9] M. Wytock, and J. Kolter, "Contextually supervised source separation with application to energy disaggregation," in Proceedings of the AAAI Conference on Artificial Intelligence, 2014.

[10] E. Vrettos, E. C. Kara, E. M. Stewart, and C. Roberts, "Estimating PV power from aggregate power measurements within the distribution grid," Journal of Renewable and Sustainable Energy vol. 11, no. 2, pp. 023707 , 2019.

[11] H. Shaker, H. Zareipour, and D. Wood, "A data-driven approach for estimating the power generation of invisible solar sites," IEEE Transactions on Smart Grid, vol. 7, no. 5, pp. 2466-2476, 2015.

[12] H. Shaker, H. Zareipour, and D. Wood, "Estimating power generation of invisible solar sites using publicly available data," IEEE Transactions on Smart Grid, vol. 7, no. 5, pp. 2456-2465, 2016.

[13] J. Bright, S. Killinger, D. Lingfors, and N. Engerer, "Improved satellitederived PV power nowcasting using real-time power data from reference PV systems," Solar Energy, vol. 168, pp. 118-139, 07/01, 2018.

[14] M. Pierro, M. De Felice, E. Maggioni, D. Moser, A. Perotto, F. Spada, and C. Cornaro, "Data-driven upscaling methods for regional photovoltaic power estimation and forecast using satellite and numerical weather prediction data," Solar Energy, vol. 158, pp. 1026-1038, 2017.

[15] F. Bu, K. Dehghanpour, Y. Yuan, Z. Wang, and Y. Zhang, "A DataDriven Game-Theoretic Approach for Behind-the-Meter PV Generation Disaggregation," IEEE Transactions on Power Systems, vol. PP, no. 99, pp. 1-1, 2020.

[16] Y. Wang, N. Zhang, Q. Chen, D. S. Kirschen, P. Li, and Q. Xia, "DataDriven Probabilistic Net Load Forecasting With High Penetration of Behind-the-Meter PV," IEEE Transactions on Power Systems, vol. 33, no. 3, pp. 3255-3264, 2018.

[17] X. Y. Zhang, S. Kuenzel, and C. Watkins, "Feeder-Level Deep Learningbased Photovoltaic Penetration Estimation Scheme," in 2020 12th IEEE PES Asia-Pacific Power and Energy Engineering Conference (APPEEC), 2020, pp. 1-5.

[18] N. S. US Department of Commerce, and N. C. f. E. I. Information Service, "NOAA National Centers for Environmental Information (NCEI)."

[19] S. Burt, "The Climatological Observers Link (COL) at 50," Weather, vol. 75 , no. 5 , pp. 137-144, 2020.

[20] I. S. Data, "National Climatic Data Center (NCDC)," Asheville, NC, 2001.

[21] C. Seger, "An investigation of categorical variable encoding techniques in machine learning: binary versus one-hot and feature hashing," 2018.

[22] A. Géron, Hands-on machine learning with Scikit-Learn, Keras, and TensorFlow: Concepts, tools, and techniques to build intelligent systems: O'Reilly Media, 2019.

[23] I. T. Jolliffe, and J. Cadima, "Principal component analysis: a review and recent developments," Philos Trans A Math Phys Eng, vol. 374, no. 2065, pp. 20150202, 2016.

[24] A. Natekin, and A. Knoll, "Gradient boosting machines, a tutorial," Frontiers in neurorobotics, vol. 7, no. 21, 2013-December-04, 2013.

[25] A. Zeyer, R. Schlüter, and H. Ney, "Towards Online-Recognition with Deep Bidirectional LSTM Acoustic Models," in Interspeech 2016, 2016.

[26] K. P. Schneider, Y. Chen, D. P. Chassin, R. G. Pratt, D. W. Engel, and S. E. Thompson, Modern grid initiative distribution taxonomy final report, Pacific Northwest National Lab.(PNNL), Richland, WA (United States), 2008.

[27] S. J. Pan, and Y. Qiang, "A Survey on Transfer Learning," IEEE Transactions on Knowledge Data Engineering, vol. 22, no. 10, pp. 13451359,2010

[28] A. Arnold, R. Nallapati, and W. W. Cohen, "A Comparative Study of Methods for Transductive Transfer Learning," in Workshops Proceedings of the 7th IEEE International Conference on Data Mining (ICDM 2007), October 28-31, 2007, Omaha, Nebraska, USA, 2007.

[29] N. Blair, A. P. Dobos, J. Freeman, T. Neises, M. Wagner, T. Ferguson, P. Gilman, and S. Janzou, System advisor model, sam 2014.1. 14: General description, National Renewable Energy Lab.(NREL), Golden, CO (United States), 2014. 